Variations sur la lettre, le mètre et la mesure :

Shakespeare

\title{
Rencontre avec Daniel Mesguich
}

Daniel Mesguich

\section{(2) OpenEdition \\ Journals}

Édition électronique

URL : http://journals.openedition.org/shakespeare/973

DOI : $10.4000 /$ shakespeare. 973

ISSN : 2271-6424

Éditeur

Société Française Shakespeare

Édition imprimée

Date de publication : 1 novembre 1996

Pagination : 81-87

Référence électronique

Daniel Mesguich, «Rencontre avec Daniel Mesguich », Actes des congrès de la Société française

Shakespeare [En ligne], 14 | 1996, mis en ligne le 01 janvier 2007, consulté le 01 mai 2019. URL : http:// journals.openedition.org/shakespeare/973; DOI : 10.4000/shakespeare.973 


\section{Variations sur la lettre,}

le mètre et la mesure

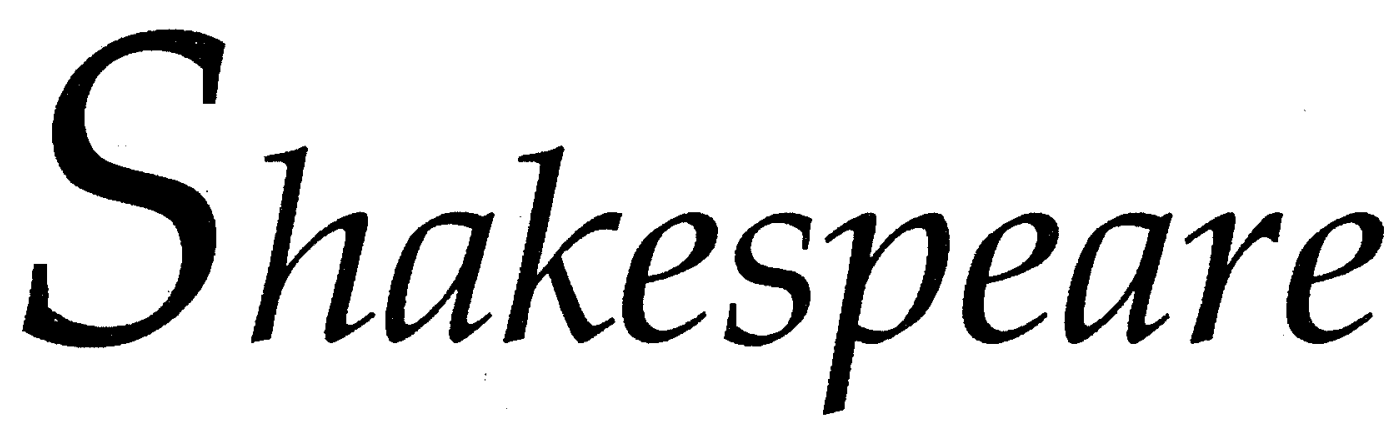

textes présentés par Dominique Goy-Blanquet

Directeur de la publication : Richard Marienstras 


\section{Rencontre avec Daniel Mesguich directeur du théâtre de (La Métaphore), Lille}

J.-M. Deprats : Depuis Hamlet en 1977, Daniel Mesguich nous a gratifié d'un Roi Lear, d'un Roméo et Juliette et plus récemment d'un Titus Andronicus qui avait suscité notre enthousiasme. Daniel Mesguich a toujours répondu à nos invitations avec bonne grâce. Aujourd'hui nous l'accueillons avec le même plaisir, le thème du Congrès nous semblant particulièrementsusceptible d'éveiller sa curiosité.

Daniel Mesguich : Oui, car au fond, toute l'œuvre de Shakespeare, en tout cas c'est ainsi que je la mets en scène, pose la question du rapport entre le présent du théâtre et son support, gravé dans le sable, sur un papier ou dans un livre, qui pourrait s'appeler "la lettre" ou "l'écrit". Pour souligner que c'est d'abord le jeu, et son plaisir, qui travaille quand je travaille les textes de Shakespeare, je voudrais commencer par faire l'acteur et vous lire un texte de Borges, "Every thing and nothing", tiré de son recueil L'Auteur :

Il n'y avait personne en lui; derrière son visage (qui, même d'après les mauvaises peintures de l'époque, ne ressemble à aucun autre) et derrière ses discours, qui furent nombreux, fantastiques et agités, il n'y avait qu'un peu de froid, un rêve que personne ne rêvait. Au début, il crut que tout le monde était comme lui, mais l'étonnement d'un ami avec qui il essaya de commenter cette vacuité, l'avertit de son erreur et lui fit comprendre pour toujours qu'il ne convient pas à l'individu de s'écarter des normes de l'espèce. Une fois, il pensa qu'il trouverait peut-être dans les livres un remède à son mal, et il apprit de cette manière ce peu de latin et cet encore moins de grec que devait mentionner un contemporain. Il considéra que la pratique d'un rite élémentaire de l'humanité pouvait bien être ce qu'il cherchait et il se laissa initier par Anne Hathaway, au cours d'une longue sieste de juin. A vingt et un ans il se rendit à Londres. Instinctivement, il s'était déjà entraîné à simuler qu'il était quelqu'un, afin qu'on ne découvrît pas sa condition d'être personne; à Londres, il identifia la profession à quoi il était destiné, celle de l'acteur, lequel, sur une scène, joue à être un autre, devant une réunion de gens qui jouent à le prendre pour cet autre. Le métier d'histrion lui apprit un bonheur singulier, peut- 
être le premier qu'il connût; mais le dernier vers declamé et le dernier mort retiré de la scène, la saveur odieuse de l'irréalité l'envahissait de nouveau. Il cessait d'être Ferrex ou Tamerlan et redevenait personne. Aux abois, il eut l'idée d'imaginer d'autres héros, d'autres fables tragiques. De cette manière, pendant que son corps s'acquittait de son destin de corps dans les bordels et les cabarets de Londres, l'âme qui l'habitait était César, qui néglige l'avertissement de l'augure, et Juliette, qui déteste l'alouette, et Macbeth, qui parle sur la lande avec les sorcières qui sont aussi les Parques. Personne ne fut autant d'hommes que cet homme qui, à la ressemblance de l'Egyptien Protée, put épuiser toutes les apparences de l'être. Parfois, il laissait dans le recoin d'une cuvre quelque confession, assuré qu'on ne la déchiffrerait pas : Richard affirme ainsi qu'en un seul personnage il joua le rôle de beaucoup, et Iago dit étrangement: "Je ne suis pas ce que je suis." L'identité fondamentale d'exister, de rêver et de représenter lui inspira des passages fameux.

Il persista vingt ans dans cette hallucination dirigée, mais il fut saisi un matin par la nausée et l'horreur d'être tant de rois qui meurent par l'épée et tant de malheureux amants qui se réunissent et se séparent et agonisent mélodieusement. Ce même jour, il décida de vendre son théâtre, il retourna dans la semaine à son village natal où il retrouva les arbres et la rivière de son enfance et il ne les rattacha pas à ces autres que sa muse avait célébrés et que rendaient illustres des allusions mythologiques et des vocables latins. Il fallait qu'il fût quelqu'un. Il fut un impresario en retraite qui avait fait fortune et qui était passionné par les prêts, les procès et la petite usure. En ces dispositions, il dicta le testament aride que nous connaissons et qui écarte délibérément tout trait pathétique ou littéraire. Des amis de Londres avaient coutume de visiter sa retraite. Il reprenait pour eux le rôle de poète.

L'histoire ajoute que avant ou après sa mort, il se sut en face de Dieu et lui dit: "Moi qui ai été tellement d'hommmes en vain, je désire en être un seul qui soit moi." D'un tourbillon, la voix de Dieu lui répondit: "Moi non plus je ne suis pas; j'ai rêvé le monde comme tu as rêvé ton œuvre, William Shakespeare, et parmi les apparences de mon rêve il y a toi, qui comme moi, es multiple et comme moi, personne. 
A la suite de Borges, sans prétendre me comparer à lui, je yais lancer des fragments - façon de parler en homme de thêâtre plus qu'en commentateur des œuvres de Shakespeare. Pour les acteurs, le "grand thêatre" est "troué" : entre la phrase "un" et la phrase "deux", il y a une quantité de phrases invisibles, qui ne se disent pas, mais qui prennent la place de l'espace possible, typographiquement, entre le point et la majuscule de la phrase suivante. Avant d'en venir à la "lettre" de l'œuvre, commençons par nous dire que ce sont des lettres trouées, ajourées, qui laissent passer la lumière. Un texte de Shakespeare n'est jamais qu'une grande lettre ou un assemblage de lettres, un livre, et à l'intérieur du livre il y a un petit livre, comme à l'intérieur du théâtre il y a un thêâtre. Au commencement était la lettre. L'écriture se transmet et se donne, semble dire Shakespeare chaque fois qu'un personnage tend un papier ou reçoit une lettre, et cela pourrait être une loi : toute pièce de théâtre qui ne se dédouble pas de cette façon à un moment ou l'autre n'est pas une grande pièce. Il le dit avec d'autres lettres que les lettres écrites, par exemple le travestissement : faire du thêâtre, c'est se déguiser, se faire prendre pour un autre pendant une durée momentanée; on se re-déguise sans cesse à l'intérieur du texte luimême.

Le "grand" thêâtre a toujours cherché, pour s'éloigner de la réalité afin de la mieux donner à lire, à se rapprocher d'elle. La fameuse règle des trois unités du thêâtre classique français traduit le désir de rappeler l'acte du théâtre : que, dans la fiction, il n'y ait qu'un seul temps, comme, dans la réalité, il n'y a qu' un temps pendant lequel se joue la représentation; un seul lieu, comme, dans la réalité, il n'y a qu' un lieu où se joue la représentation; une seule action, comme, dans la réalité, il n'y a qu' une action, celle de jouer la représentation. Comme. La règle des unités mime le présent du thêâtre. La manière de Shakespeare est plus forte, plus folle. Pas de parallélisme. C'est bien dans l'acte même du thêâtre que se joue une grande partie de l'intrigue. Ou plutôt, ce qui est "intrigue" et ce qui nous intrigue, ce n'est pas tellement tout ce qu'on met derrière ce nom - fable, anecdote, histoire, flux narratif, diégèse, contenu de l'œuvre - mais au-delà de ce qui est raconté, mille choses qui se divisent de plus en plus infiniment.

Une lettre n'est lisible pour nous qu'à partir du moment où on la reconnaît, sinon elle n'existe pas. Shakespeare nous somme d'être kabbalistes. Dans ses pièces, quoi qu'elles racontent, tout est signe de quelque chose à découvrir. Je vois un mot, une phrase et j'entends deux, trois sens. Hamlet a failli me rendre fou : Ophélie, ce doit être "orpheline", mais le "r" est tombé; ou bien "ob helios", "trop proche du soleil", le soleil représente le père dans les théories freudiennes, quels sont les rapports avec Polonius ? Et ainsi de suite, sans fin. Est-ce juste ou faux ? Ni l'un ni l'autre. La question n'est plus là. La question est que "le travail est un trésor": plus vous chercherez, plus vous trouverez. Le texte majoritaire de Shakespeare n'est pas celui qui est écrit.

Prenez les lettres qui s'échangent dans les textes : celle que Titus Andronicus envoie aux dieux, le livre où Lavinia fait découvrir son malheur, le billet de Shylock, etc... Autant de signaux qui renvoient au fait que tout cela était écrit, qui renvoie encore à autre chose, à ces trous, ces vides, ces silences. Presque toutes les pièces de Shakespeare possèdent un morceau de texte qui se dit comme texte. A 
quoi sert-il ? à révéler, à dévoiler, parfois à dévoyer, comme dans Roméo et Juliette, où la lettre arrive trop tard. Quand le spectre apparait sur les remparts, Hamlet l'écoute, puis se précipite sur ses tablettes pour noter ce qu'il a entendu. Si le spectre de mon père apparaissait dans ma chambre ce soir, je ne penserais pas : "Je vais oublier, vite, il faut que j'écrive ce qu'il vient de me dire", je crois que je m'en souviendrais. Selon la psychanalyse, on écrit pour se débarrasser. Et c'est ce que fait Hamlet avec son propre père. Le petit texte qu'écrit Hamlet sur la scène, seul et devant tout le monde, reste un secret. Là encore, ce facétieux Shakespeare montre que, ce qui est d'abord à lire n'est pas le spectre mais le reste, tout ce qui ne semble pas se donner à lire, qui s'écrit à même le corps, à même les personnages. Et quelle est cette écriture qui ne s'écrit pas à l'intérieur du texte ? C'est la tragédie même. On peut l'entendre dans un sens fataliste : c'était écrit, c'était le destin.

Un acteur a trois positions devant la phrase qu'il doit jouer. Si j'existe avant la phrase, ce n'est pas ce que je dis qui me forme. Je peux être pendant ma phrase, m'y promener comme un explorateur qui pousse des lianes en faisant attention où poser le pied, et je n'existerai que par cette phrase qui m'érige en sujet. Ou je peux être après ma phrase; quoi que je fasse, elle est là avant moi et je ne suis plus que le canal qu'elle traverse. Shakespeare joue avec ce troisième cas de figure. Le "c'était écrit", tout à coup, jaillit comme un cri, comme un muscle blanc. Les lettres ne sont pas les seuls signes d'antériorité. Roméo et Juliette se passe à Vérone, Titus Andronicus, à Rome. S'agit-il vraiment de Vérone, de Rome ? Non, c'est une façon de colorier ce qui précèderait et qui serait impossible à dire. En effet, cette écriture recèle des choses impossibles à dire ou écrire, et comme l'inspecteur de police parsème une poudre sur les objets pour révèler les empreintes digitales, Vérone est la poudre, un lieu, un temps, impossibles à conceptualiser. La langue elle-même y échouerait puisqu'elle toucherait là un trou du texte et non pas le texte. Pensez à tous les grands écrivains, les philosophes, qui ont peiné sur "l'être", "le non-être". Shakespeare se contente d'une phrase et chacun de dire que c'est profond, mais en fait c'est profond parce que c'est vide, cela produit un appel d'air. En paraphrasant légèrement Lévinas, ce n'est pas ce que veut dire le texte qui compte. Non seulement il y a des trous entre la phrase "un" et la phrase "deux", mais à l'intérieur même de la phrase "un" ou "deux", il y a encore des trous que Shakespeare nous invite à remplir. Et là-dessus personne n'arrive à se mettre d'accord : le fameux livre que porte Hamlet à la main, c'est tantôt L'Etre et le néant, tantôt c'est Le Capital, de Karl Marx, tantôt, La Bible. Chaque fois on cite un autre ouvrage et chaque fois on a raison, parce que le livre est vide. Il existe juste assez pour articuler des plis - le silence - dans le blanc de la page; on entend les plis, les blancs du silence. Et les mots ne demandent qu'une chose, c'est littéralement d'être dépliés, expliqués. Autrement dit, il n' y a rien à lire, circulez. Mais c'est parce qu'il n'y a rien à lire qu'on ne circule pas et qu'on cherche à s'approcher de plus en plus prêt. Saint Augustin disait qu'il y a trois temps : le présent du passé, le présent du présent et le présent du futur. C'est-à-dire qu'il n'y a toujours qu'un temps. Mais le plus fort c'est de nous le dire au XVIIe siècle et qu'au XXe siècle, on s'échine encore à l'apprendre. Voilà le "c'était écrit" dont je parlais tout àl'heure. 
$Q u$. : Pensez-vous que représenter Shakespeare à Stratford, bien établi en bourgeois prospère à la fin de sa vie, comme l'a fait Edward Bond, était une entreprise aussi légitime que de monter ses cuvres?

D. M. : Il y a des écrivains positifs, "pleins" - cela ne veut pas dire qu'ils sont mauvais, mais leur texte n'est pas ajouré, il risque de tomber d'autant plus vite. Ce qui défie le temps et le dépasse, c'est ce qui ne rentre pas dans la réalité. L'erreur de Shylock, c'est de vouloir "la lettre et rien que la lettre", et vouloir qu'elle s'incarne, mais justement le théâtre n'incarne pas. Que Shakespeare continue à avoir existé ou pas, à être ou ne pas être, qu'il clignote, c'est encore du sens. Bond a rempli les blancs à sa manière, comme si momentanément, de manière imparfaite, non finie, il coloriait le dessin. C'est légitime, à condition que cela tombe tout de suite. Si sa pièce devait mettre un point final à l'énigme Shakespeare et le faire incarner une fois pour toutes, ce serait lamentable. Il faut l'entendre comme une œuvre de journaliste, ce qui n'empêche pas Bond d'être un grand écrivain.

Qu. : Ne pourrait-on pas imaginer Shakespeare dans un temps qui n'existe pas : le plus-que-présent?

$D . M$. : J'ai écrit un texte sur le plus-que-présent au théâtre. Les grandes pièces sont toujours philosophiques, elles s'ouvrent sur des questions. Quand on croit avoir mis un point final, ce n'était qu'un point de suspension, de nouveau il y a un effondrement de terrain du sens et l'on repart. Les textes de Shakespeare tournent autour de trois ou quatre questions, qui elles-mêmes se subdivisent. Il ne faut pas confondre l'acteur et le personnage, mais la première réplique de Hamlet est: "Qui va là ?". Qui sait ? Qu'est le sujet, le temps, le centre ? Chaque fois, la réponse est le terme de la question : le qui, le quoi, le quand. Parfois, quelques mots de question jaillissent comme des morceaux de confessions, comme pour exciter la curiosité. On annonce à Laertes qu'Ophélie est morte et aussitôt, il demande : "Où ?" Vous trouvez cela normal ? En fait oui, il a raison, c'est une question fondamentale : où est la mort, où est le meurtre ? Shakespeare relie toutes sortes de fils qui sont autant de mythes. On mentionne toujours le mythe d'CEdipe, évidemment, parce que Freud l'a rendu célèbre, mais on peut aussi bâtir une théorie psychanalytique sur le mythe de Hamlet. On voit des noms qui tournent, des glissements de sujets : Polonius prend la place de Claudius dans la chambre de Gertrude et c'est pour cela qu'il meurt, Claudius a pris la place de Hamlet père, Hamlet père avait sans doute pris la place du père de Fortimbras, Rosencrantz a pris la place de Guildenstern, tous les deux ont pris la place de Horatio, Horatio a pris la place de Polonius puisque c'est lui qui raconte, Laertes prend la place de Hamlet, etc... Et ça tourne, ça tourne, tous ces fills sont indicateurs d'une chose impossible : le fameux centre vide dont je parlais tout à lheure. Shakespeare ne le remplit pas, il pose des questions, il ne donne pas les réponses. Il dit que le spectre apparaît sur le rempart d'Elseneur à minuit. Le réali sateur de cinéma croit que c'est vrai et cherche un château où placer sa caméra avec un acteur recouvert d'un drap blanc en extérieur nuit. Ce "rempart" marque la limite entre l'intérieur et l'extérieur, entre Elseneur et ce qui n'est pas Elseneur. C'est une 
frontière comme la rampe, entre la scène et la salle. De même le "spectre", c'est le spectacle, spectarer, spéculer, c'est l'œil, l'apparition donc - spectacle-spectre, rampe-rempart. Le spectre arrive, il est et il n'est pas, il est aussi la frontière entre la présence et l'absence, comme minuit est une frontière entre un jour et un autre, entre un règne et un autre. Autrement dit, Shakespeare annonce que la pièce commence "entre deux choses", le château n'est qu'une façon de colorier. Il existe un théâtre qui traduit le cinéma. Shakespeare parle de rempart, et un certain théâtre - Brook n'en est pas loin - fait un grand praticable qui voudrait dire "château", "rempart", mais qui ne dit rien du tout, sinon qu'on n'a pas de château, alors on va vous faire un truc en bouts de bois qui voudra dire "château". C'est du cinéma en pauvre, ce n'est pas du théâtre. Si on fait descendre un ciel de deux mètres d'ouverture, c'est la traduction d'un vrai ciel au cinéma. Mais si tout d'un coup on y pénètre, on y disparaît, alors ce n'est plus le ciel, c'est une façon de figurer l'infigurable, de faire voir l'invi sible et cela devient du théâtre. Il ne s'agit pas de traduire mot à mot mais ouvrir sur autre chose, c'est ce que nous dit Shakespeare : "Je vais vous montrer ce que c'est maintenant, tout de suite. Je vous ai préparé un morceau de présent déjà imbibé de passé, voire de projection de futur. A vous de jouer. Demandez-vous qui vous êtes, demandez-vous ce qu'est votre temps." Si la pièce nous montrait Vérone ou le XVIIe siècle, elle serait un documentaire, mais si maintenant elle nous dit qui je suis, où je suis, quand je suis, ce qu'est le présent, cela devient du Shakespeare.

$Q u$. : Shakespeare a été traduit dans la plupart des langues du monde, pourtant on entend souvent dire qu'il est encore à traduire, et qu'il faut être écrivain pour cela. En français, André Gide s'était entouré de conseillers, pourtant il a commis des faux-sens et des contre sens. Qu'en pensez-vous?

D. $M$. : Celui dont on peut dire qu'il n'est pas encore traduit, son œuvre continue, comme le vin, de mieux en mieux. Shakespeare a continué son ouvre en nous laissant cette énigme - ai-je existé ou non ?. Le texte original est en anglais, mais êtes-vous sûr que c'est l'original ? Savez-vous ce qu'est l'origine ? Les personnages de théâtre disent "j'existe" pour donner figure à ce que l'on ne peut pas voir, l'origine qui échappera toujours. Quand Michel Vitoz a traduit Hamlet, d'une manière un peu archaïsante, tout en jouant beaucoup sur le XXe siècle, nous affirmions que le texte que avait été écrit par Guillaume Branlelance, dont Shakespeare n'était que le traducteur anglais. Guillaume Branlelance a existé, pas plus pas moins que Shakespeare ou que Timon d'Athènes. Ce ne sont que des jalons, des rôles, des personnages pour faire passer du sens et de l'énigme. Croire au texte original, ce serait croire qu'e Hamlet existe en chair et en os, même si un roi a pu s'appeler ainsi quelque part dans un lointain passé. Mais cela nous est égal. Même les pièces historiques ne sont pas historiques, ou à peine plus que les autres, le Henry VI sur la scène n'a rien à voir avec le roi du même nom. Le texte original non plus n'est pas incarné, lui aussi, il est l'énigme d'un autre texte. Cette genèse infinie ne nous mène nulle part, on ne trouvera pas un point d'émission qui serait enfin l'original. Ce qu'on appelle Shakespeare ce sont simplement ces voyages. 
Y. Thoret : Quand j'ai vu votre mise en scène d'Anne Boleyn, je me demandais si ce ne serait pas d'actualité de monter Othello pour parler de cette obscurité, cette absence de certitudes?

$D . M$ : A présent, je dis beaucoup moins qu'avant, que je voudrais monter l'intégrale de Shakespeare.

R. Gardette Votre discours sur le théâtre justifie la création du "théâtre du miroir", avec l'utilisation du miroir au cœur de l'expérience théâtrale.

D. M. : C'était le nom de ma compagnie, qui est devenue le théâtre de (La Métaphore), car notre miroir était une métaphore. Le miroir et ses doubles, disait-on dans la presse. J'en ai beaucoup joué parce que Shakespeare le fait sans arrêt. Chaque personnage est le double imparfait de lui-même : le même mais l'un à midi, l'autre à 16 heures; l'un jeune, l'autre vieux; maigre ou gros; blond, brun; autre mais pas non plus radicalement autre. A l'époque, je lisais Freud et aussi Fred, un auteur de bande-dessinée dont le héros s'appelle Philémon. Quand Philémon se regardait dans un miroir la main en l'air, le miroir renvoyait une image qui se grattait le nez comme il l'avait fait précédemment, alors il appelait le réparateur de miroirs...Nous avons pris le nom de "théâtre du miroir" en imaginant un miroir un peu détraqué. Chaque personnage déteint un peu sur l'autre, produit un écho décalé. Les frontières sont mises en jeu parce qu'elles ne sont pas sûres, il n'y a pas d'assurance que Hamlet ne soit pas Laertes, ou Gertrude, ou Hamlet père, etc... La pièce brasse un sujet unique, littéralement scié en plusieurs. C'est une mise en étoile de ce qui semble un sujet global, totalisé, plein - et brusquement voilà d'autres pistes qui s'ouvrent, il faut s'y mettre à plusieurs, parfois engager vingt acteurs pour répondre à la question "qui ?" Le vers est coupé à l'hémistiche, il pourrait y avoir un hémistiche de l'être coupé en son milieu. On laisse chanter les deux morceaux.

M. Jones-Davies : Dans son "Sermon sur la mort", Bossuet reprend une phrase de Tertullien qui dit que le cadavre est sans nom. Cet excès de lettres, tous ces signes qu'on imprime sur le corps n'ont pour but que de combler ce vide insup portable. Le désir de mettre des mots sur le cadavre que nous devenons revient à ce que vous disiez sur le théâtre qui ne représente que des spectres, c'est le trou sans nom que l'on essaye de remplir. Mais on met tellement de lettres sur le corps qu'on finit par le tuer.

D.M. : Nous acteurs, nous nous impliquons dans la lecture physiquement, ce que nous donnons à lire est aussi notre corps en train de le lire. Le théâtre est une étrange activité parce que tout ce qui s'écrit se donne à lire et tout ce qui se donne à lire est écrit; cela peut se décrire, mais tout n'est pas écrit. Une mise en scène ne s'écrit pas parce qu'elle est trop ancrée - et encrée - dans le présent. 